\title{
Emergent functional behavior of humic substances as result of chemical characteristics
}

\author{
Vialykh E.A. ${ }^{1,2}$ \\ ${ }^{1}$ University of Colorado, Boulder, CO, USA, elena.vialykh@colorado.edu \\ ${ }^{2}$ BioHumaNetics Inc. Gilbert, AZ, USA
}

doi: 10.36291/HIT.2019.vialykh.049

The structural organization of humic substances (HS) has been a central question of earth sciences for several decades. Recently, HS were recognized as complex mixtures of small molecules and oligomers. We investigate the correlation between the chemical composition of HS, perceived as labile aggregates, and their emergent functions (EF).

Complex organic mixtures can be considered within the context of complexity theory. The study is done through investigations of how interactions between parts creates an emergent collective system behavior and how the system interacts and forms relationships with its environment [1]. In order to create a model of HS, which illustrates the connection between chemical composition and EF, the theoretical model should encompass properties that relate to $\mathrm{H}$-bonding, hydrophobic and charge-transfer interactions and depend on conformations. Therefore, we developed a computational approach that combines with ideas of supramolecular structural organization and dynamic combinatorial chemistry [2]. The focus of the work was on the labile (reversible and changeable), connection between molecular units with bonds (interactions) that form and break on elementary step timescales. The important reactions are dissociation, proton transfer, hydrogen bond interactions, which occurs in a timescale range of pico- to nanoseconds. Therefore, the composition of a system will depend on its environment.

Computational modelling was used to help to understand the processes and mechanisms on the molecular scale that occur in different fractions of the HS, fulvic acids and humic acids, as they interact with metal ions and organic pollutants. The conformational changes in different solvents were also investigated. The importance of non-covalent interactions in the EF of HS is highlighted. H-bonding, hydrophilic/hydrophobic surface areas and $\pi$-stacking interactions play a significant role in aggregation processes as well as in the sorption of environmental pollutants. The interaction of a polar pollutant, phenol, with HS occurs through $\mathrm{H}$-bonding, whereas non-polar benzene interacts through hydrophobic and m-stacking interactions. The non-polar pollutant results in a much stronger sorption by HS and causes an additional structural rearrangement of the aggregates. The interactions of HS with metal ions revealed a variation of the studied properties of $\mathrm{HS}$, including $\mathrm{H}$-bonding, structural conformation, the hydrophilic/hydrophobic surface areas and the potential energy, with the change of environmental conditions. Thus, a decrease of water content or an increase of cation concentration resulted in higher Me binding and aggregation with a decrease of the potential energy, i.e. formation of more stable aggregates.

The significance of this work is in shifting the focus from a search for the exact "unique" structure of HS to the correlation of chemical characteristics of the sample with its emergent functional behavior. The new approach could be beneficial in solving ongoing disputes in the field of HS research, since it reveals how different properties of HS emerge from their chemical composition. Overall in this work we showed that recognition of HS as labile supramolecular aggregates of different molecular fragments, allows one to consider the lability of the system when it interacts with environmental pollutants.

References

1. Bar-Yam Y. https://www.researchgate.Net/Publication/246294756_General_

Features of Complex Systems (2002).

2. Corbett P.T. et al. // Chem. Rev. 2006. 106:3652. 